\title{
Tunable emission in dye-doped truxene-based organogels through RET
}

\author{
Juan F. Galisteo-López*†, Sandra Gómez-Esteban ${ }^{\mathrm{a}}$, Berta Gómez-Lor ${ }^{\mathrm{a} *}$, Cefe López $^{\mathrm{a}}$ \\ ${ }^{a}$ Instituto de Ciencia de Materiales de Madrid, c/Sor Juana Inés de la Cruz 3, 28049 \\ Cantoblanco (Madrid). Spain
}

\begin{abstract}
:
Organic systems comprising a truxene-based organogel doped with an organic dye have been fabricated and their photophysical properties examined in the search for an organic matrix with tunable luminescent properties. The addition of the organic dopant has been observed to introduce changes in the morphology of the gel which alters the ratio between monomer and excimer species. Further, the luminescent properties of the doped organogel have been studied and their evolution with dopant concentration explained in terms of resonant energy transfer between the excimer species (acting as donor) and the organic dopant (acceptor). The interplay between blue, green and red emission bands associated with monomer, excimer and organic dopant allows tuning the luminescence of the system within the visible reaching white light emission under certain conditions. The origin of the energy transfer is found to be the aggregation of the molecules upon solvent evaporation, being the extreme case the more stable xerogel phase which constitutes a technologically relevant approach where solvent evaporation is not an issue.
\end{abstract}

* juan.galisteo@csic.es, bgl@icmm.csic.es

$\uparrow$ Present address: Instituto de Ciencia de Materiales de Sevilla (ICMSe-CSIC), c/Américo Vespucio 49, 49092 (Sevilla). Spain 


\section{Introduction}

The ability of certain organic molecules to self-assemble in solution into onedimensional (1D) superstructures via directional interactions (hydrogen bonding, $\pi$ stacking, etc.) has attracted considerable interest over the past two decades. These 1D structures can further intertwine forming an interpenetrated network that eventually immobilizes solvent molecules triggering the gelation of the solvent. The fibrillar morphology of such organogels or their solvent-free xerogel counterparts, are of interest in applications ranging from organic electronics ${ }^{1}$ to tissue engineering ${ }^{2}$ or switchable molecular traps. ${ }^{3,4}$

While a deterministic choice of molecules to gelate a given solvent, and thus create an organogel, is not straightforward due to the lack of clear design principles to induce gelation $^{5}$ their potential is widely acknowledged. In particular, their supramolecular structure has attracted interest from the point of view of light-harvesting architectures where energy transfer from the organogel to a dopant molecule acting as an acceptor can take place. After initial seminal work in the early $2000 \mathrm{~s}^{6,7}$ a large number of organogels have been explored as platforms for efficient energy transfer ${ }^{8,9}$ with particular interest in the design of materials with tunable luminescent properties and eventually white light emission. $^{10,11,12,13}$

Among the different low molecular weight organogelators for light harvesting, those forming $1 \mathrm{D}$ stacks of $\pi$-extended molecules have attracted most attention, as such $\pi$ stacked assemblies provide efficient pathways for energy migration. Truxene is a highly stable heptacyclic $\pi$-conjugated platform with promising semiconducting ${ }^{14,15}$ and strong blue light emitting properties ${ }^{16}$ which derivatives, when conveniently functionalized, are prone to form organogels. ${ }^{17,18}$ Particularly interesting for optoelectronic applications are truxene derivatives functionalized with long alkyl chains in 2,7 and 12 positions, that are able to gelate varied solvents due to their high tendency to form columnar superstructures with high aspect ratio, promoted by solvophobic and $\pi$ - $\pi$ stacking interactions. In this work we profit from the gelating properties of a truxene-derivative based organogel 2,7,12-tris(dec-1-ynyl)-10,15-dihydro-5H-diindeno[1,2-a:1',2'c]fluorene ${ }^{18}$ to fabricate an organic matrix with tunable luminescent properties by doping it with an organic dye Rhodamine $\mathrm{B}(\mathrm{RhB})$. In this system blue and green emission (arising from monomer and excimer species of the organogel) can be combined with a red component provided by the organic dopant to obtain a broad photoluminescence (PL) chromatic palette. The possibility of tuning the PL of the system is demonstrated by controlling the degree of aggregation (and therefore the ratio of monomer/excimer emission) which induces resonant energy transfer (RET) from the donor supramolecular 
framework to the acceptor molecule $\mathrm{RhB}$ by varying the dopant concentration. The colorimetric properties of both, the gel and xerogel phases, are monitored as the dopant organic dye concentration is varied and the possibility of achieving white light emission is demonstrated. Further, evidence for structural changes taking place in the supramolecular system as a consequence of dye doping are presented.

\section{Experimental}

\section{Sample preparation}

In a standard procedure $22.1 \mathrm{mg}$ of the truxene derivative shown in Fig. 1a were added to $1 \mathrm{~mL}$ of cyclohexane. The mixture was heated at $60^{\circ} \mathrm{C}$ until homogenization took place. After cooling it at room temperature for several minutes the organogel formed over the entire sample, evidenced by the fact that no flowing of the solution was observed as the cuvette was turned upside down.

Doped gel samples were prepared by addition of $10 \mu \mathrm{L}$ of a $\mathrm{RhB}$ solution $(0.0,0.01,0.05$, $0.1,0.2,0.5 \mathrm{~mol} \%$ ) in chloroform to previously formed gels, then we repeated the process for gel formation.

\section{Optical characterization}

PL spectra were obtained pumping with the third harmonic $(\lambda=355 \mathrm{~nm})$ of a pulsed $\mathrm{Nd}$ :YAG laser delivering 9 nanosecond long pulses with a repetition rate of $10 \mathrm{~Hz}$ and a pump energy of $30 \mathrm{~mJ}$. Spectra were collected with a fiber coupled spectrophotometer (Ocean Optics USB4000). Time resolved measurements were carried out with a Time Correlated Single Photon Counting (TCSPC) card (Becker \& Hickl) using a pulsed laser source (OPerA-Solo from Coherent) delivering $150 \mathrm{fs}$ long pulses with a $\lambda=355 \mathrm{~nm}$ and a repetition rate of a $1 \mathrm{kHz}$.

\section{Results and discussion}

Truxene-based organogels were fabricated according to Ref. 18 in quartz cuvettes (see Experimental). In an initial stage the emission properties of the organogel were studied (see Fig. 1). Under UV ( $\lambda=355 \mathrm{~nm}$ ) excitation the gel presents a PL spectrum with two well separated bands. The structured (featureless) band centered at $425 \mathrm{~nm}(530 \mathrm{~nm})$ corresponds to the monomer (excimer) species in the gel. The presence of the two bands points to the coexistence of both species, contrary to observations in solution where only the monomer is present. ${ }^{18}$ Though both bands have similar intensity, the fraction of excimer species is much larger than that of the monomer as the quantum yield (QY) of the former is expected to be much lower than the latter in this kind of systems. ${ }^{19} \mathrm{PL}$ 
decay measurements were also performed at wavelengths close to the band center for each species, showing a multiexponential behavior of the decay curves. Further, a longer lifetime associated with the excimer was observed (see Fig. S1 and details in the supplementary information), expected for systems where the dissociation of the excimer does not occur within the lifetime of the monomer, as in the case of our gel. ${ }^{20}$
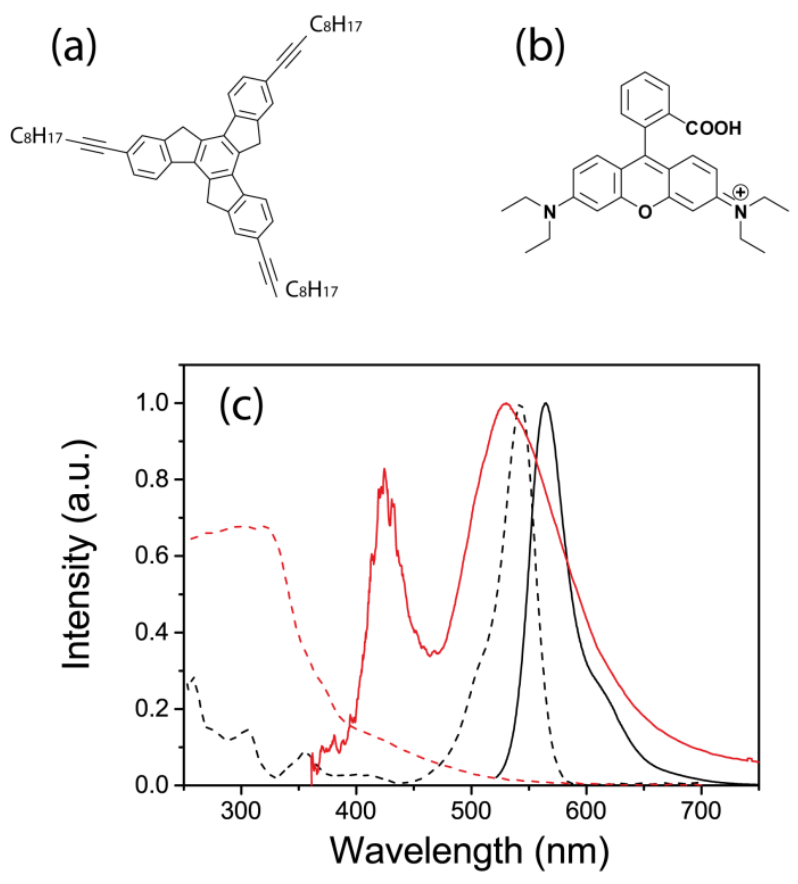

Fig. 1 (a) and (b) show the chemical structure of the truxene derivative and RhB. (c) PL (solid) and absorption (dashed line) of the organogel (red) and RhB (black line).

Under prolonged optical pumping, the organogel presented drastic changes in its PL (see Fig. 2). While the excimer component of the PL spectrum remained constant in time, the monomer one dropped by more than $40 \%$ of its initial value. This is a local effect at the region of irradiation, apparent to the naked eye as a dark spot coinciding with the pump spot, which recovered after leaving the gel a few minutes without illumination (see below). Both, the PL dynamics as well as the recovery time depend on the irradiation conditions. Variations of the PL due to fluctuations in the pump were ruled out as the pump power was monitored during the experiment and checked to be constant within $5 \%$, way below changes observed for the monomer species.

The above changes in PL cannot be due to a photobleaching of the molecules since they recover their emissive properties after a few minutes. A plausible cause is the 
introduction of morphological changes due to a local heating at the pumped region. Local disruption of the gel due to melting can be also ruled out as the monomer component of the PL in solution is the predominant one (see above), contrary to what we observe here. The changes in the PL spectra can however be explained as a local evaporation of the solvent which would favor aggregation and therefore the presence of excimer species. This hypothesis was corroborated by forming a xerogel upon drying the gel in the cuvette evaporating the solvent at room temperature. Fig. S2 in the supplementary information shows how the monomer PL band in the extreme case of the xerogel, where no solvent is left, is now much lower than the one associated with the excimer. This point will be discussed later. The fact that no change is observed in the excimer PL upon a strong decrease of the monomer emission can be understood in the light of the above mentioned fact that the QY of the former is well below that of the latter: a small decrease in the fraction of monomer species (and a corresponding increase in the fraction of excimers) would induce larger changes in the PL of the species with larger QY.
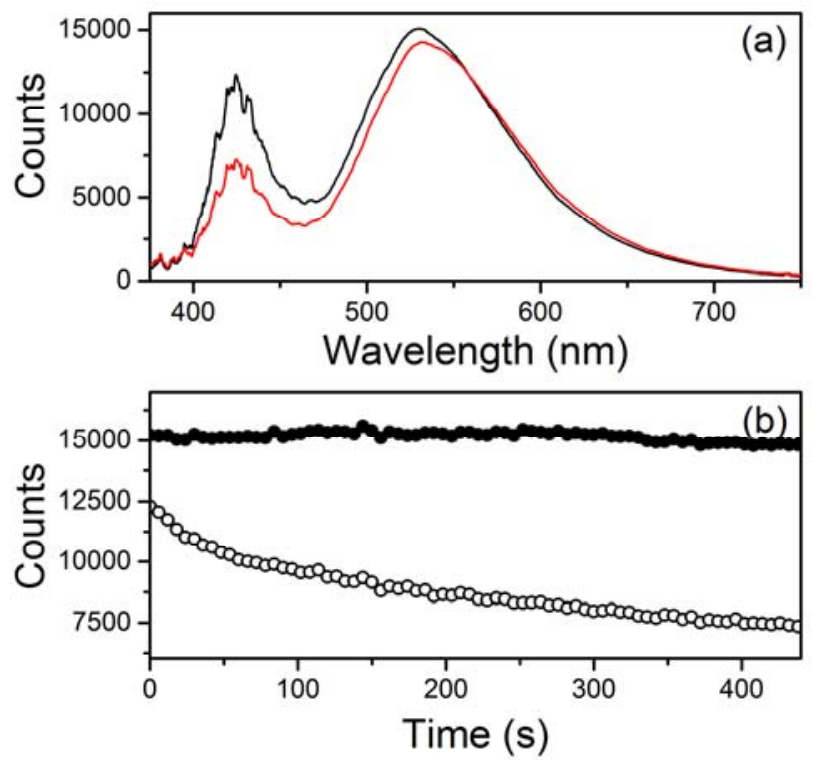

Fig. 2 (a) PL spectra of an organogel before (black) and after 7 minutes of UV irradiation (red curve). (b) Evolution of monomer (open) and excimer (solid dots) PL peak intensity under UV irradiation.

In order to provide the organogel PL, comprising "blue" and "green" monomer and excimer bands, with a "red" component we added RhB to the sol prior to gelation. This organic dye has an absorption band which matches the gel's excimer emission and can thus form an acceptor/donor pair for energy transfer (see Fig. 1). Upon addition of RhB, a number of changes take place in the photophysical properties of the gel. First of all, a 
ca. ten-fold enhancement in the PL associated with the monomer species takes place which is visible even by the naked eye. Fig. 3 shows gels with different amounts of RhB under VIS (3a) and UV (3b) illumination, where a clear blue color originating from monomer PL is evident upon RhB addition. On the contrary, nearly no change is present for wavelengths corresponding to the excimer band (see Fig. S3).
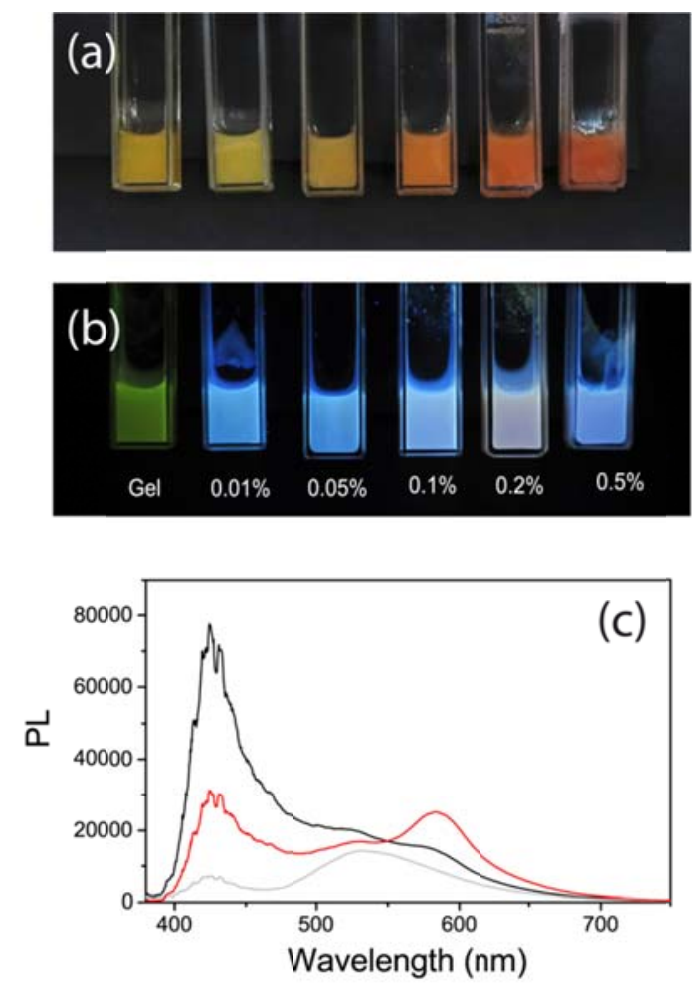

Fig. 3 Gels with different amounts of RhB under ambient (a) and UV (b) illumination. (c) PL spectra of gel containing $0.2 \mathrm{~mol} \% \mathrm{RhB}$ before (black) and after (red curve) 10 minutes of pumping with $\lambda=355 \mathrm{~nm}$. Grey curve shows the PL spectrum of a gel containing no dye.

Apparently the highly polar dopant is producing an important effect on the aggregation tendency of these molecular units, favoring the presence of monomeric species. It has been previously shown that aggregation of this compound is highly dependent of the polarity of the environment. ${ }^{18}$ Comparison of the SEM images of the xerogels obtained with and without RhB (see Fig. S4) clearly shows how the addition of the dye decreases the entanglement of the fibers confirming a disrupting effect on the aggregation exerted by the dopant. In order to discard a possible effect of the dopant in the packing mode of molecules within the fibers, the X-ray diffraction pattern of a xerogel containing 0.2 mol\% RhB and a xerogel without $\mathrm{RhB}$ were compared. Both patterns are identical demonstrating that the presence of the dye in the sample does not influence the mode the molecule self-assembles (see Fig. S5 in the supplementary information). 
Figure $3 \mathrm{c}$ shows spectra of the above mentioned samples before and after UV irradiation for the case of a gel containing $0.2 \mathrm{~mol} \% \mathrm{RhB}$. We can see how after ca. 10 minutes of UV pumping (under the same conditions mentioned before), the monomer PL decreases and a PL peak centred at 580nm associated with RhB appears. This can be better appreciated in Fig. S3 (see supplementary information) where spectra for samples with a range of dye concentrations are shown. Results for the sample with the largest RhB load $(0.5 \mathrm{~mol} \%)$ are not shown as it did not dissolve properly resulting in an inhomogeneous optical response. As we increase the amount of dopant added to the gel the different components of the PL (monomer, excimer and dopant) undergo a different behavior (see Fig. S3c-e). After seven minutes of illumination, when the PL has reached a stationary behavior, we can see how increasing the dopant concentration has a small effect on the monomer and excimer which oscillate (20\% and $8 \%$ in amplitude respectively) around a constant value. Such oscillations can be due to small inhomogeneities of the dopant distribution which brings small changes in aggregation. On the contrary, the PL peak associated with the dopant emission increases monotonically with concentration.

The presence of a PL peak associated with $\mathrm{RhB}$ is clearly related to the above mentioned structural changes that the organogel undergoes upon illumination. Dynamic measurements monitoring the PL of the three species: monomer, excimer and RhB simultaneously (see Fig. S6) show how a drop in monomer emission (associated with solvent evaporation as already discussed) is accompanied by an enhancement of RhB PL. Since RhB could not be directly excited with the pump wavelength (away from the RhB absorption band), the latter points to $\mathrm{RhB}$ (acting as an acceptor molecule) being excited through RET from excimer species (acting as donor) once the solvent is evaporated and donor-acceptor distance is reduced. Radiative transfer from the excimer to the RhB molecules is ruled out as it should not depend on the distance between both species and hence should already be present before solvent evaporation.

The above mentioned dynamics have the same local character as for the bare gel, a dark spot appearing at the pump region which vanishes after a few minutes without illumination, coinciding with the recovery of the PL of the gel. Fig. S7 in the supplementary information shows the time evolution of the PL at three different wavelengths matching the emission of monomer, excimer and $\mathrm{RhB}$ of an organogel containing $0.2 \mathrm{~mol} \%$ before and after a 7 minute period without illumination. It can be appreciated how after removing the UV illumination the PL signal recovers to ca. $80 \%$ of its initial value for both, the monomer and the RhB.

Colorimetric data extracted from the above spectra confirm the shift from green to blue in the gel PL as RhB is added to the gel (Fig. 4a). Further, after UV illumination we can 
see how the drop in monomer emission and the onset in RhB PL modify the colorimetric properties of the multicomponent system making it possible to yield white light for a $\mathrm{RhB}$ concentration as low as $0.2 \% \mathrm{wt}$ (Fig. $4 \mathrm{~b}$ ).
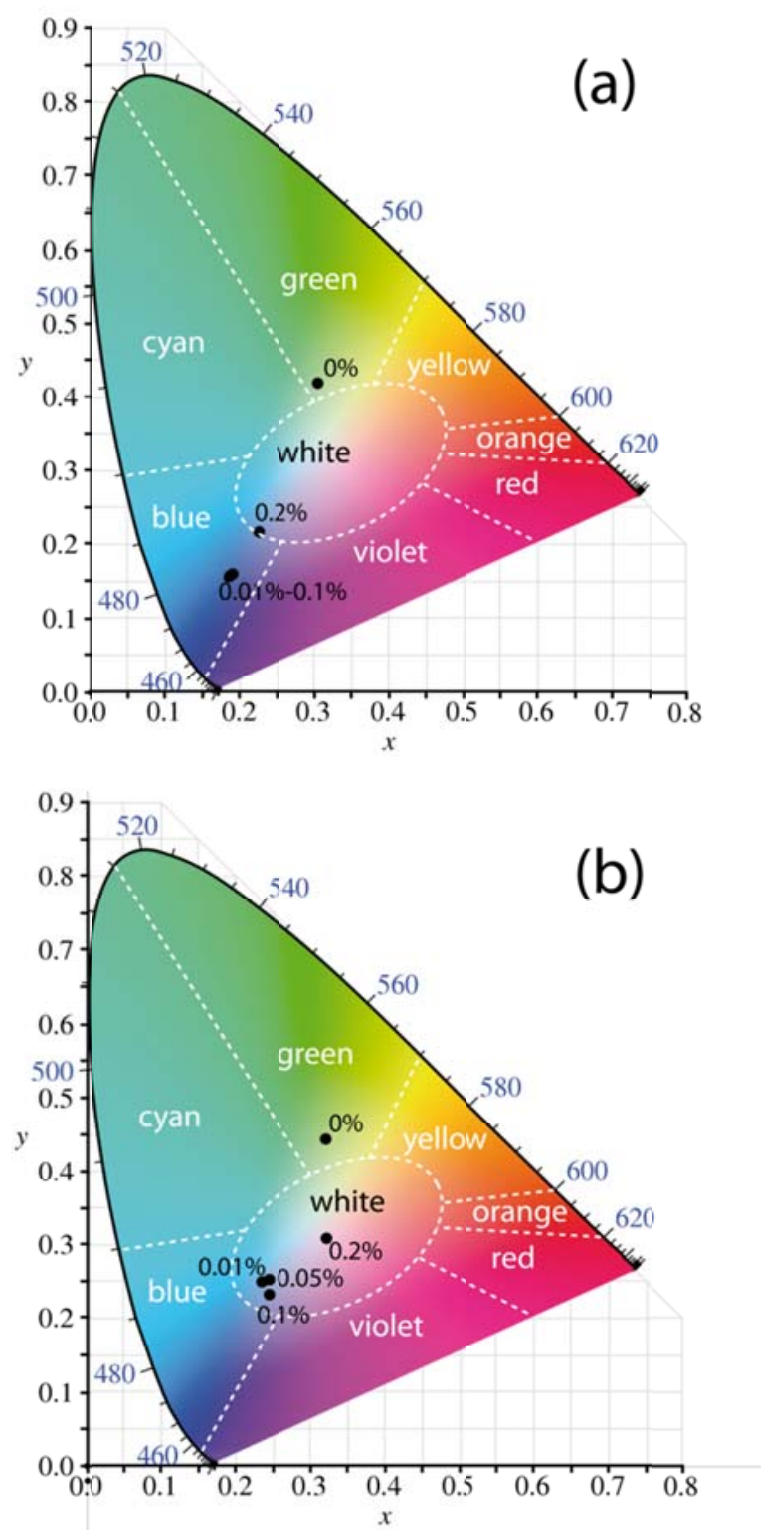

Fig. 4 CIE 1931 chromaticity diagram for the emission of organogrels with different amounts of RhB as grown (a) and after seven minutes of $U V$ illumination (b).

While experimental evidences mentioned above indicate that the excitation mechanism of RhB is non-radiative, a conclusive evidence of which type of RET ${ }^{20}$ (Förster, Dexter,etc.) has not been yet achieved. PL decay measurements as those in Fig. S1 were carried out to check whether faster dynamics for the excimer took place, the addition of non-radiative paths to the de-excitation of the excited donor being the hallmark of RET. Though no changes were 
observed with respect to the system without $\mathrm{RhB}$, this does not rule out RET as the amount of acceptor $(\mathrm{RhB})$ molecules is much smaller (ca. 3 orders of magnitude) than that of donor (truxene) molecules. Hence the fraction of excimers acting as donors is too small to be detected in a time-resolved measurement. This point also explains the fact that upon addition of $\mathrm{RhB}$, the associated PL peak centred at 580nm appears without the monomer emission undergoing a noticeable reduction.

The fact that white light emission takes place as the solvent evaporates locally is relevant from the point of view of applications as issues related with solvent evaporation, detrimental for devices, could be avoided. With this in mind we considered the photophysical properties of the xerogels, obtained by evaporating the solvent from the organogels and forming a thin film on the wall of the quartz cuvette. Fig. 5 shows spectra of xerogels with and without the addition of $\mathrm{RhB}$. Spectra have been normalized to the excimer emission band for comparison.

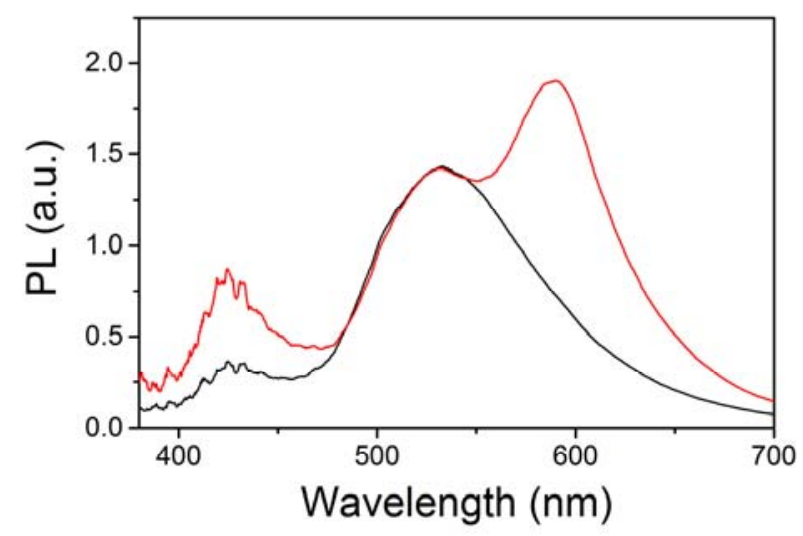

Fig. 5 PL spectra of a bare xerogel (black) and a xerogel containing $0.1 \mathrm{~mol} \%$ of $\mathrm{RhB}$ (red curve).

For the xerogel obtained from the bare gel the PL is dominated by excimer emission as mentioned above. If the gel contains $\mathrm{RhB}$ two main differences are observed in the xerogel upon solvent evaporation. On the one hand, the monomer emission band increases relative to the excimer one. Such enhanced monomer emission confirms disruption of aggregation effected by the doping dye already seen in the case of the gel. On the other hand a PL band associated with RhB appears at 580nm evidencing RET as the donor and acceptor species come into close vicinity when the gel collapses. The combination of both effects, taking place gradually as we increase the amount of $\mathrm{RhB}$ in the gel (see Fig. S8 in the supplementary information), provides a means to tune the system PL. If we consider the ratio of monomer/excimer PL, we can see how it rises with small amounts of RhB and then slightly decreases (see Fig. S8b), indicating that the 
xerogel disruption depends on the $\mathrm{RhB}$ concentration. On the other hand, the ratio between RhB/excimer PL, giving an estimate of the RET efficiency (as no direct excitation of the RhB takes place as already mentioned), monotonically increases withas we add RhB (see Fig. S8c).

In a colorimetric plot (see Fig. 6) we see a transition from the characteristic green emission of the xerogel, dominated by the excimer PL, to chromatic values within the white region upon addition of small amounts of $\mathrm{RhB}$ and finally reaching yellow-green as the RhB PL increases. Finally contrary to what was observed in the gel, no changes on the xerogel PL were observed upon prolonged (over 10 minutes) UV irradiation, further supporting the hypothesis that the observed dynamic changes in xerogel emission were due to the local evaporation of the solvent.

For the monomer species we have extracted a QY value of 0.31 from PL measurements in dilute solutions of the truxene molecules. While measurements in the solid phases (gel and xerogel) will be needed in the future if applications in solid state lighting are envisaged, the value obtained for the monomer should be considered as an upper limit of the system as excimer QY is expected to be lower. ${ }^{19}$ At this point a compromise between QY and a straightforward solution process manufacturing should be made.

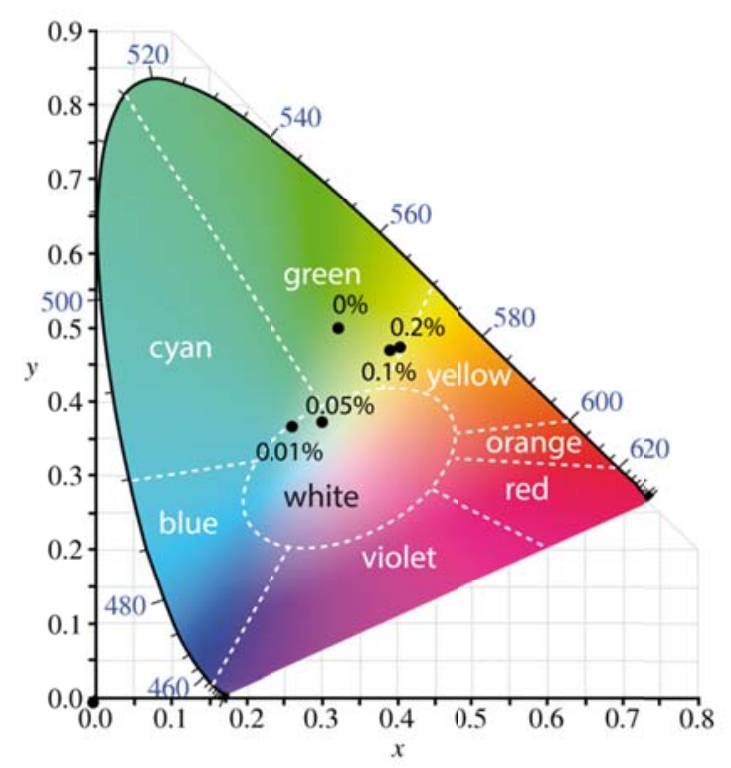

Fig. 6 CIE 1931 chromaticity diagram showing the position of xerogels $P L$ with different amounts of $R h B$. 


\section{Conclusions}

In summary, we have studied the photophysical properties of truxene-based organogels doped with organic dye RhB. Very low amounts of dopant were observed to strongly modify the morphology of both the gel and the xerogel, altering the ratio between monomer and excimer species. Further, evidence for RET between the excimer species (acting as donor) and $\mathrm{RhB}$ (acting as acceptor) has been observed in the xerogel which behaves as a light harvesting system with tunable luminescent properties in the visible region of the electromagnetic spectrum. While preliminary results have been presented with a red emitting acceptor, changing the emission properties of the dopant could boost the palette of achievable PL. Further such versatile luminescent properties in a solventfree, and thus technologically relevant, system could be suited for future applications dealing with solid state lighting.

\section{Acknowledgements}

This work was partially funded by EU FP7 NoE Nanophotonics4Energy grant No. 248855; the Spanish Government MINECO MAT2012-31659 (SAMAP) and CTQ201340562-R grants; and the Comunidad de Madrid S2013/MIT-2740 (PHAMA_2.0) and CAM S2013/MIT-2740 projects.

\section{Notes and references}

Electronic Supplementary Information (ESI) available: [details of any supplementary information available should be included here]. See DOI: 10.1039/b000000x/

1 S. S. Babu, S. Prasanthkumar and A. Ajayaghosh, Angew. Chem., 2012, 51, 1766.

2 K.Y Lee and D.J. Mooney, Chem. Rev., 2001, 101, 1869.

3 H. Xu, S.P. Stampp and D.M. Rudkevich, Org. Lett., 2003, 5, 4583.

4 H. Xu, D.M. Rudkevich, Org. Lett., 2005, 7, 3223.

5 R. G. Weiss. J. Am. Chem. Soc. 2014, 136, 7519.

6 T. Sagawa, S. Fukugawa, T. Yamada and H. Ihara, Lanmguir 2002, 18, 7223.

7 T. Nakashima and N. Kimizuka, Adv. Mater., 2002, 14, 1113.

8 A. Ajayaghosh, V. K. Praveen and C. Vijayakumar, Chem. Soc. Rev., 2008, 37, 109. 
9 V. K. Praveen, C. Ranjith, E. Bandini, A. Ajayaghosh and N. Armaroli, Chem. Soc. Rev., 2014, 43, 4222.

10 R. Abbel, R. van der Weegen, W. Pisula, M. Surin, P. Leclère, R. Lazzaroni, E.W. Meijer and A. P. H. J. Schenning, Chem. Eur. J. 2009, 15, 9737.

11 C. Vijayakumar, V. K. Praveen, A. Ajayaghosh, Adv. Mater., 2009, 21, 2059.

12 C. Giansante, G. Raffy, C. Schäfer, H. Rahma, M-T. Kao, A. G. L. Olive, A. Del Guerzo, J. Am. Chem. Soc. 2011, 133, 316.

13 For a recent review see: V.K. Praveen, C. Ranjith, N. Armaroli, Angew. Chem.Int. Ed., $2014, \mathbf{5 3}, 365$.

14 Y. M. Sun, K. Xiao, Y.Q. Liu, J.L. Wang, J. Pei, G. Yu, D.B. Zhu, Adv. Funct. Mater. $2005, \mathbf{1 5}, 818$.

15 K.-Q. Zhao, C. Chen, H. Monobe, P. Hu, B.-Q. Wang, Y. Shimizu, Chem. Commun. $2011,47,6290$.

16 A.L. Kanibolotsky, R. Berridge, P.J. Skabara, I.F. Perepichka, D.D.C. Bradley, M. Koeberg, J. Am. Chem. Soc. 2004, 126, 13695.

17 K.-P. Tseng, M.-T. Kao, T. W. T. Tsai, C.-H. Hsu, J. C. C. Chan, J.-J. Shyue, S.-S. Sun, K.-T. Wong, Chem. Commun. 2012, 48, 3515.

18 S. Gómez-Esteban, M. Pezella, A. Domingo, G. Hennrich, B. Gómez-Lor, Chem. Eur. J. 2013, 19, 16080.

19 “ $\pi$-stacked polymers and molecules", T. Nakano (Ed.) (Springer Japan, 2014).

20 “Molecular fluorescence”, B. Valeur (Wiley-VCH, 2001). 